\title{
The significance of pancytopenia in miliary tuberculosis
}

\author{
Beverley J. Hunt, Vivienne Andrews and K.W. Pettingale \\ Departments of Medicine and Haematology, King's College Hospital, London SE5, UK.
}

\begin{abstract}
Summary: Patients with miliary tuberculosis accompanied by a pancytopenia rarely survive their disease. If the peripheral blood picture does recover it has been taken as an indication that there is no underlying haematological disease, and so re-examination of the bone marrow is not performed.

A case is described where resolution of the pancytopenia occurred but a persisting haematological disease remained. Aspects of haematological disease associated with tuberculosis are discussed.
\end{abstract}

\section{Introduction}

There are many haematological states associated with tuberculosis especially miliary tuberculosis. The majority of patients have little disturbance in their haematology other than the normochromic or hypochromic, normocytic anaemia of chronic disease. The degree of anaemia tends to reflect duration of illness rather than the severity. A raised erythrocyte sedimentation rate (ESR) and blood viscosity are also common; the ESR may be over 100 especially in cryptic miliary tuberculosis. ${ }^{1}$ White cell counts are usually slightly reduced or within normal limits with a left shift' (increase in premature forms). ${ }^{2}$ Frank leucopenia occurs infrequently while leucocytosis and thrombocytopenia ${ }^{3}$ have been described and thrombocytosis can occur as part of the 'reactive' changes.

Uncommon red cell changes include sideroblastic anaemia and also megaloblastic anaemia which is usually due to folate deficiency as B12 levels may be high due to liver involvement or occasionally low due to concomitant pernicious anaemia. ${ }^{4}$ Folate levels are frequently reduced, reflecting the poor physical condition and poor dietary intake of the patient. Rarely, secondary malabsorption occurs due to ileocaecal tuberculosis.

Anti-tuberculous drugs may alter the haematological picture due to idiosyncratic side effects. Rifampicin may cause an immune haemolytic anaemia and/or thrombocytopenia, by producing rifampicin dependent antibodies while PAS can behave in a similar way and also causes hypothrom-

Correspondence: B.J. Hunt, M.R.C.P., North London Blood Transfusion Centre, Deansbrook Road, Edgware, Middlesex HA8 9BD, UK.

Accepted: 24 March 1987 binaemia. Many drugs such as pyrazinamide produce a sideroblastic anaemia, whilst idiosyncratic episodes of agranulocytosis or even aplastic anaemia have occurred especially with streptomycin. The Committee on the Safety of Medicines have had 59 reports of blood dyscrasias with 6 fatalities associated with antituberculous drugs between $1964-85 .^{5}$

A higher incidence of tuberculosis in haematological disease is disputed, whilst disseminated disease is unarguably commoner, especially with haematological malignancies. ${ }^{6,7,8}$ Disseminated disease is similarly more frequent in other immunosuppressed states such as infection with the human immunodeficiency virus. ${ }^{9}$ Disseminated atypical mycobacterial infections are associated with a high incidence of haematological abnormalities although it is uncertain that the primary disease is the infection in these patients. ${ }^{10,11}$

Areactive miliary tuberculosis occurs mainly in patients with haematological disease. ${ }^{7}$ Oswald ${ }^{8}$ found only 4 of 120 patients with reactive miliary tuberculosis had a serious blood disorder unlike 6 out of 8 with areactive miliary tuberculosis. It is characterized by little tissue reaction to the infection but enormous numbers of acid-fast bacilli are present, reflecting failure of cell-mediated immunity.

The diagnosis of tuberculosis must be entertained in all patients with unexplained fever who have haematological disease and/or immunodeficiency and especially in the elderly where cryptic miliary tuberculosis is difficult to diagnose. In these situations a bone marrow and liver biopsy are most useful for histology and culture. ${ }^{1}$

A variety of abnermal haematological pictures is described as part of, or dominating the presenting

(C) The Fellowship of Postgraduate Medicine, 1987 
clinical picture in tuberculosis, especially with disseminated disease. ${ }^{12}$ These include leukaemoid reactions, myelofibrotic changes, the haemophagocytic syndrome,$^{13}$ polycythaemia and pancytopenia. ${ }^{14}$ The main problem in assessing these reports is deciding whether the patients had tuberculosis superimposed on a blood disease or whether tuberculosis does occasionally produce a clinical picture similar to leukaemia or myelofibrosis. The answer remains difficult to elucidate and it is assumed if the patient recovers from the haematological abnormality with anti-tuberculosis treatment this strongly suggests the abnormality is secondary to the infection.

Rarely tuberculosis presents with pancytopenia. Cooper ${ }^{15}$ describes a patient where pancytopenia resolved with the removal of an enlarged tuberculous spleen. Otherwise pancytopenia attributable to tuberculous hypersplenism has rarely been seen. ${ }^{16,17}$ Splenomegaly is uncommon in tuberculosis; $13 \%$ of Munt's series had mild splenomegaly ${ }^{17}$ but only 1 in 68 patients and 3 of 24 post-mortems in Biehl's series ${ }^{18}$ of cases of miliary tuberculosis.

If a patient presents with pancytopenia and tuberculosis and treatable causes of pancytopenia are excluded, does this pancytopenia reflect an underlying haematological disorder or is the pancytopenia solely secondary to the reported myelosuppressive effects of tuberculosis? Most reported cases have underlying haematological disease, ${ }^{12,19}$ but the second hypothesis has been supported by cases where recovery of peripheral blood counts with anti-tuberculous therapy has been accepted as evidence of a normal bone marrow. ${ }^{20,21} \mathrm{~A}$ patient presenting with pancytopenia and tuberculosis is described below, where the peripheral blood count returned to normal and tuberculosis resolved on treatment but a haematological disorder persisted.

\section{Case report}

A previously fit 71 year old Caucasian woman complained of 6 months' general malaise with anorexia, weight loss, weakness, headaches and ankle swelling for 2 weeks. She was teetotal, a non-smoker and took occasional paracetamol. On admission she looked ill, was anaemic and pyrexial but with no organomegaly. A blood count showed haemoglobin $6.0 \mathrm{~g} / \mathrm{dl}$, white blood cells $0.9 \times 10^{9} / 1$, (differential : neutrophils $68 \%$, lymphocytes $32 \%$ ) platelets $60 \times 10^{9} / 1$, ESR $110 \mathrm{~mm} /$ hr. A bone marrow aspirate was a bloody tap but a bone marrow biopsy showed focal hyperplasia and abnormal megakaryocytes with a moderate increase in reticulin fibres. A chest X-ray exhibited heavily calcified mediastinal lymph nodes with diffuse shadowing in the lung fields. Biochemical analysis showed hyponatraemia and raised liver enzymes; serum protein electrophoresis showed a monoclonal IgG kappa band $(5.9 \mathrm{~g} / \mathrm{l})$ with otherwise normal immunoglobulins, urine contained Bence Jones protein (kappa). Sucrose lysis and Ham's test were negative; no urinary haemosiderin was detected. Viral studies, including parvovirus, were negative.

The patient was transfused and over the next fortnight her platelet and white cell count returned to normal. However she remained unwell with a swinging pyrexia and increasingly raised liver enzyme levels including a serum B12 of $1,114 \mathrm{ng} / 1$ (normal 80-800). Computed tomographic (CT) scan of chest and abdomen showed enlarged para-aortic lymph nodes and a small opacity at the base of the left lung. Epithelioid and giant cell granulomata with incipient central necrosis and acid-fast bacilli were found on a liver biopsy. Subsequently, previously collected early morning urine samples grew Mycobacterium tuberculosis and granulomata were seen on a further bone marrow biopsy.

Appropriate anti-tuberculosis therapy was given for 9 months and the markers of her illness including the pyrexia returned to normal, while the peripheral blood count was normal throughout. Bone marrow biopsies taken 6 and 18 months after her illness and review of original histology showed myelodysplastic changes of increasing severity, with abnormal localization of immature precursors ${ }^{22}$ and increase in reticulin fibres although the peripheral count still remained norma

She remained well for a year but then gradualle became pancytopenic again without evidence recurrence of tuberculosis. At present she is transfusion dependent; the bone marrow shows more severe myelodysplastic changes with increased reticulin.

\section{Discussion}

The pathogenesis of pancytopenia when associated with tuberculosis has intrigued physicians and pathologists for years. In the majority of reported cases an underlying haematological disease, usually malignant, ${ }^{12,19}$ is self evident, and yet there are some cases in which the issue is unclear, so it is still cited in standard textbooks that miliary tuberculosis can produce pancytopenia. Experimental support for this was derived from the classical experiments of Sabin et al. ${ }^{23}$ who demonstrated that animals with disseminated tuberculosis involving the marrow developed pancytopenia. The hypothesis has been further supported by cases where the recovery of peripheral blood counts with anti-tuberculous therapy is taken to indicate there is no underlying haematological disease.

Patients with pancytopenia with tuberculosis have a high mortality, but there are a few that have recovered. ${ }^{14,19,20,21,24}$ Documentation of the survivors is not ideal; isolation of the acid-fast bacilli is often difficult, in some cases bacteriological proof of mycobacteria is lacking or yet in others follow up was 
inadequate. Some have not recovered their peripheral count fully, e.g., a persisting leucopenia; others have subsequently manifested a frank haematological malignancy; Katsen described a patient who recovered from pancytopenia and bone marrow necrosis to reveal Philadelphia positive chronic myeloid leukaemia. ${ }^{19}$ Mangion $^{21}$ described the only documented case where tuberculosis was proven and a pancytopenia recovered fully and the patient was followed for 9 months, although some may dispute that the patient fulfills the definition of pancytopenia as the lowest white cell count was only $4.0 \times 10^{9} / 1$. As in other cases recovery of the peripheral count was taken to indicate there was no underlying haematological disease so bone marrow histology was not repeated after completion of treatment. Our case disputes this supposition as the subsequent clinical course and bone marrow biopsies reveal a persisting haematological disorder despite a normal peripheral count. Furthermore resolution of pancytopenia occurred independently of anti-tuberculous treatment. The transient nature of the peripheral pancytopenia in this patient probably reflects decompensation of a failing but just compensating marrow after the added insult of miliary dissemination, with blood transfusion relieving the

\section{References}

1. Proudfoot, A.T. Cryptic disseminated tuberculosis. $\mathrm{Br} J$ Hosp Med 1971, 773-780.

2. Sahn, S.A. \& Neff, T.A., Miliary tuberculosis Am J Med 1974, 56: 495-505.

3. Kalinowski, S.Z. \& Walker, J.M. Thrombocytopenic purpura in tuberculosis. Brit J Tuberc 1956, 50: 239.

4. Coburn, R.J., England, J.M., Samson, D.M. et al. Tuberculosis and blood disorders. Br J Haematol 1973, 25: 793-799.

5. CSM Update. Br Med J 1986, 293: 11631.

6. Lowther, C.P. Leukaemia and tuberculosis. Ann Intern Med 1959, 51: 52.

7. O'Brien, J.R. Non-reactive tuberculosis. J Clin Path 1954, 7: 216.

8. Oswald, N.C. Acute tuberculosis and granulocytic disorders. Br Med J 1962, ii: 1489.

9. Pitchenik, A.E., Cole, C., Russell, B.S. et al. Tuberculosis, atypical mycobacteriosis and A.I.D.S. among Haitians and non-Haitian patients in South Florida. Ann Intern Med 1984, 101: 641-645.

10. Zamorano, J. \& Tompsett, R. Disseminated atypical mycobacteria; infection and pancytopenia. Arch Intern Med 1968, 121: 424-427.

11. Gordon-Smith, E., Holt, J.M. Areactive generalized tuberculosis as a diagnostic problem in clinical haematology. $Q J$ Med 1967, 36: 602.

12. Glasser, R.M., Walker, R.I. \& Herion, J.C. The significance of haematological abnormalities in patients with tuberculosis. Arch Intern Med 1970, 125: 691 - 695.

13. Weintraub, M., Siegman-Ingra, Y., Josiphov, J., Rahmani, R. \& Liron, M. Histiocytic haemophagocytosis in stress.

In patients with pancytopenia and miliary tuberculosis, the underlying haematological disease is often leukaemia or frequently a pre-leukaemia which today, as with this case, is classified within the myelodysplastic syndromes. This patient had a non-progressive monoclonal gammopathy as is often seen in patients with myelodysplasia. ${ }^{26}$

If pancytopenia reflects an underlying pre-existing haematological disease it is likely that the loss or diminution of the normal immune response permits the reactivation of old tuberculosis lesions or a new opportunistic infection. ${ }^{4}$ Active tuberculous infection has a prevalence from 4.6 to $12.8 \%$ in post-mortems of leukaemic patients. ${ }^{6,8,25}$ Variations in host response or infecting organism are seen in this group of patients, they may be areactive, i.e., there is a lack of tissue response, ${ }^{7}$ or an infection with atypical mycobacteria. ${ }^{10}$

In conclusion we suggest that if a pancytopenia occurs with miliary tuberculosis it reflects an underlying haematological disorder. Peripheral film and bone marrow review is essential if the patient recovers with anti-tuberculous treatment even if the peripheral count is normal.

miliary tuberculosis. Arch Intern Med 1984, 144: 20552056.

14. Medd, W.E. \& Hayhoe, F.G. Tuberculous miliary necrosis with pancytopenia. $Q J$ Med $1955,96: 351$ - 363 .

15. Cooper, W., Pancytopenia associated with disseminated tuberculosis. Ann Intern Med 1959, 50: 1497-1501.

16. Meredith, H.C., Early, J.Q. \& Becker, W. Tuberculous splenomegaly with the hypersplenism syndrome. Blood 1949, 4: 13671.

17. Munt, R.W. Miliary tuberculosis in the chemotherapy era: with a clinical review in 69 American adults. Medicine 1972, 51: 139.

18. Biehl, J.P. Miliary tuberculosis. Am Rev Respir Dis 1958, 77: 605 .

19. Katzen, H. \& Spagnolo, V. Bone marrow necrosis from miliary tuberculosis. JAMA 1980, 244: 2438-2439.

20. Mangion, P.B. Disseminated tuberculosis complicated by pancytopenia. Proc $R$ Soc Med 1971, 64: 1000.

21. Rosenberg, M.J. \& Rumans, L.W. Survival of a patient with pancytopenia and disseminated coagulation associated with miliary tuberculosis. Chest 1978, 73: 536-539.

22. Tricot, G., Boogaerts, M.A., De Wolf-Peeter, C. et al. The myelodysplastic syndromes: different evolution patterns based on sequential morphological and cytogenetic investigations. Br J Haematol 1985, 59: 659-670.

23. Sabin, F.R., Doan, C.A. \& Forkner, C.E. Studies on tuberculosis. J Exp Med 1930, supp 13: 1-152.

24. Paulley, J.W. Blood changes associated with disseminated tuberculosis. Br Med J 1954, 411.

25. Muller, G.L. Clinical significance of blood in tuberculosis. 
The Commonwealth Fund, New York 1943.

26. Mufti, G.J., Figes, A., Hamblin, T.J., Oscier, D.G. \& Copplestone, J.A. Immunological abnormalities in myelodysplastic syndromes. $\mathrm{Br} J$ Haematol 1986, 63: 143-147. 